\title{
LOS FACTORES QUE CONFORMAN EL MODELO DE ACREDITACIÓN POR ALTA CALIDAD DE PROGRAMAS ACADÉMICOS EN COLOMBIA, REVISIÓN DESDE EL ENFOQUE DE LA PERCEPCIÓN
}

\author{
THE FACTORS THAT CONSTITUTE THE MODEL OF ACCREDITATION FOR \\ HIGH QUALITY OF ACADEMIC PROGRAMS IN COLOMBIA, \\ REVIEW FROM THE APPROACH OF PERCEPTION
}

RECIBIDO: Agosto 20 de 2013

ACEPTADO: Octubre 30 de 2013

\section{RESUMEN}

El objetivo del presente artículo de investigación consistió en conocer la percepción respecto a los factores que conforman el modelo de acreditación de alta calidad en programas académicos universitarios en Colombia, de dos grupos de expertos representados por pares y los directivos académicos que hayan participado en procesos de esta naturaleza. La metodología desarrollada tiene un enfoque cualitativo, y han sido contemplados los métodos de investigación fenomenológico y hermenéutico. Se acudió a la entrevista focalizada y la revisión documental como técnica de recolección de la información. Los resultados indican que los expertos tienen una percepción positiva del modelo de acreditación y de sus factores, éstos piensan que es indispensable revisarlo y contemplar

\footnotetext{
* Directora del Programa de Administración de Empresas de la Universidad Simón Bolívar, sede Barranquilla, Líder del grupo de investigación Gestión organizacional. auribe@unisimonbolivar.edu.co
} 
cambios en su estructura. Se concluye que existe una crítica generalizada por parte de los expertos a los factores incluidos en el modelo, por considerarlos extensos y descontextualizados, y por asumir que estos responden más a las orientaciones de organismos externos que a las necesidades propias de la calidad en un programa académico.

Palabras clave: Percepción, modelo, acreditación, organización, calidad.

\author{
ABSTRACT \\ Desarrollo Gerencial, Vol. 5-2. No. 2-pp. 21-54 julio-Diciembre Universidad Simón Bolívar-Barranquilla - Colombia, ISSN: 2145-5147 \\ .http://portal.unisimonbolivar.edu.co:82/rdigital/desarrollogerencial/index.php/desarrollogerencial/issue/archive
}


The purpose of this research was to know the perception of two groups of experts represented by pairs and the academic managers, regarding the factors that constitute the model of accreditation for high quality in academic programs of the universities in Colombia. The methodology developed has a qualitative approach, and the phenomenological and hermeneutical research methods have been considered. The focus interview and the documentary review were the techniques used for data collection. The results obtained suggest that although the experts have a positive perception of the model of accreditation and its factors, they think that it is essential to revise it and to consider changes in its structure.It is concluded that there is a generalized opinion of the experts in relation to the factors included in the model. This is because they are extensive and out of context; and it is assumed that they more likely respond to the guidelines of external organisms, than to the proper needs of quality standards in an academic program.

Key words: Perception, model, accreditation, organization, quality 


\section{INTRODUCCIÓN}

En el marco de la educación superior y como consecuencia de las decisiones del mercado, la acreditación por alta calidad se ha convertido en uno de los factores de competitividad más importantes, y en uno de los criterios de decisión y preferencias de su objetivo público. Esto se debe a que la acreditación pretende dar respuesta a las expectativas de formación de la sociedad y a las exigencias de satisfacción del cliente.

Los procesos de acreditación de universidades y programas académicos se han inspirado en los modelos de calidad aplicados en las empresas privadas, y es así como contemplan la medición de una serie de estándares, características e indicadores, de cuyos resultados depende la obtención de dicha acreditación. No obstante, por parte de las comunidades académicas, se han generado interrogantes acerca de si la calidad en un proceso educativo es susceptible de ser medida a través de esquemas similares a los que se aplican en una empresa productiva.

En este artículo, se presentan resultados parciales de una investigación que pretende conocer la percepción de los actores relacionados con los procesos de acreditación. Por tanto, en él se revisan específicamente los factores que conforman el modelo actual de acreditación a la luz de las percepciones expresadas por los expertos en el tema, como lo son los pares académicos y los directivos de programas que se han sometido previamente a estos procesos. 
Las preguntas de investigación que generan el trabajo son:

¿Cuál es la percepción por parte de los expertos acerca de los factores que conforman el modelo vigente de acreditación por alta calidad para programas académicos universitarios en Colombia?

¿Qué factores adicionaría o eliminaría con respecto al proceso de acreditación por alta calidad de programas actualmente aplicado en Colombia?

De acuerdo con lo anteriores planteamientos, el principal objetivo de investigación presentado en este artículo pretende evidenciar la percepción de los expertos, quienes tienen directa relación con los procesos de acreditación por alta calidad de programas académicos universitarios colombianos. En segunda instancia, se pretende establecer si existen en el modelo elementos que no han sido tenidos en cuenta y que podrían coadyuvar a dar una mayor integralidad al proceso, a la luz de las percepciones encontradas. Entendemos por expertos a dos clases de actores: los pares académicos que verifican las condiciones de calidad de programas y los directivos de programas académicos que se han sometido a procesos de acreditación por alta calidad.

En la estructuración del marco teórico, se examinó el concepto de percepción, como fenómeno social, antropológico y organizacional, que puede ser utilizada como herramienta para estudio de fenómenos organizacionales como el de la acreditación, a fin de entenderlo y de contextualizarlo, y a sus actores como sujetos de percepción. 
Asimismo, se revisaron las diferentes conceptualizaciones sobre organización, estrategia y cambio, y algunas teorías organizacionales afines, como la de las anarquías organizadas, los sistemas flojamente acoplados y el nuevo institucionalismo, en tanto referentes teóricos de formas organizacionales que se pueden asimilar a una universidad.

En el artículo, se presentan, en fin, resultados parciales de la investigación referentes a las percepciones encontradas en el grupo de actores representados por directivos y pares académicos de programas académicos universitarios en Colombia, con quienes se tuvo contacto directo mediante la técnica de la entrevista focalizada.

\section{MÉTODO}

La investigación que origina el artículo se concibió bajo el paradigma cualitativo. Se entiende como metodología cualitativa al "Conjunto de prácticas interpretativas que hacen al mundo visible, lo transforman y lo convierten en una serie de representaciones en forma de observaciones, anotaciones, grabaciones y documentos; es naturalista, porque estudia los objetos y los seres vivos en sus contextos naturales e interpretativo pues intenta encontrar sentido a los fenómenos" (Hernández Sampieri y otros, 2008 p. 9).

Se ha optado por esta metodología en atención a los postulados de Taylor y Bogdan (1987), quienes afirman que la metodología cualitativa se refiere a la investigación que produce datos descriptivos, a las 
propias palabras de las personas, habladas o escritas, y a las conductas observables. El enfoque cualitativo se orienta, además, bajo los postulados de Zemelman (1998), para quien este permite que la investigación sea flexible y propicia la posibilidad de que el problema de estudio se reformule en razón de las circunstancias. Los anteriores referentes metodológicos justifican el abordaje de la percepción de un proceso de acreditación por alta calidad bajo este paradigma cualitativo, por la necesidad de conocer, entender, describir, interpretar y analizar los contextos y personajes que intervienen en un proceso humano, como es la acreditación de un programa académico universitario y el análisis de los factores que lo conforman.
En este trabajo, se han considerado dos métodos de investigación: el fenomenológico y el hermenéutico. El primero se justifica por el interés de indagar y comunicar la realidad del fenómeno de la acreditación; el segundo, permite estudiarlo e interpretarlo.

Según Husserl (1995), el método fenomenológico consiste en examinar contenidos a través de la conciencia y en determinar si estos son reales o imaginarios. Pero, como se ha reiterado, la investigación también es concebida bajo el método hermenéutico, en razón a que acude a la comprensión como acontecer del sentido; bajo este método, la comprensión se constituye en el modo de ser y de estar, permitiendo reflexiones y haciendo posibles la 
interpretación y comprensión de textos (Ferraris, 2000).

Para el desarrollo del trabajo, se utilizaron como técnicas la entrevista focalizada y la revisión documental. Según Benney y Hughes (1970), las entrevistas son "la herramienta de excavar" favorita de los sociólogos, y en nuestro caso, permitieron echar mano de relatos verbales. Por sus características específicas, la categoría de la entrevista focalizada también permitió revisar previamente la situación objeto de la entrevista (Valles, 1997).

Con respecto a la revisión documental, Cázares considera que esta permite acceder a una panorámica acerca de la información relevante de diversas fuentes confiables sobre un tema específico, sin necesidad de aprobar u objetar alguna idea o postura. Para efectos del presente artículo, son presentados los resultados de acuerdo con la información obtenida a través de dos grupos de expertos: pares y directivos académicos.

En cuanto al tamaño de la muestra, Mertens (2005) plantea que este no se fija a priori, sino según unidades de observación o análisis, y se fija un número conforme se adiciona información. Dado el carácter cualitativo de la presente investigación, el tamaño de la muestra fue fijado por conveniencia, de modo que el investigador seleccionó libremente el número de los participantes según dos criterios: que tuviesen conocimiento de los procesos de acreditación, y disposición para atender a la demanda de información. También se 
tuvo en cuenta una premisa metodológica subyacente, según la cual el número de sujetos de la muestra se define por el criterio de suficiencia comparativa (Orozco, 1994), el cual exige contar con un numero de entre 12 y 25 sujetos para ser comparados entre sí. El conocimiento obtenido no pretendió tener validez estadística ni generalizarse cuantitativamente.

\section{MARCO TEÓRICO}

\section{La percepción desde el contexto organizacional}

Siendo la percepción el enfoque bajo el cual se estudian en esta investigación los criterios de los expertos frente al fenómeno de la acreditación, es importante asumirla como un hecho válido para estudiar fenómenos que se producen en las organizaciones. La percepción, en efecto, es un fenómeno humano que ha sido objeto de innumerables intentos de explicación. En los comienzos del siglo $\mathrm{XX}$, era entendida como el resultado de procesos corporales como la actividad sensorial. En ese entonces, el énfasis investigativo se centró en su caracterización psicológica; no obstante, como se ha dicho, la percepción es un hecho válido para estudiar fenómenos que se producen en las organizaciones, puesto que permite comprender la cultura, las reglas y nociones, que son patrones de conducta observables en toda empresa (Geerz, 1996).

Algunos teóricos, como Gibson (1990), se oponen a la idea de que la percepción sea del dominio de la psicología, por considerar que esta no toma en cuenta la interacción que 
establecen las personas en su

entorno cultural al buscar

información, y, en cambio, la

conciben como un proceso por el cual

el individuo da significado al ambiente

para integrar la información que

recibe con elementos del

conocimiento de las situaciones,

como las presunciones básicas de lo

que es el mundo y con los modelos

ideales, a fin de construir el mundo

que nos rodea.
La percepción puede entonces ser utilizada para el estudio de los fenómenos que ocurren en una organización, y se convierte en un proceso por el cual los individuos organizan las impresiones que recogen del ambiente con el fin de darle un sentido al entorno.

En la siguiente tabla, se establecen las diferencias entre los enfoques que estudian la percepción.

Tabla 1. Diferencias entre los enfoques que estudian la percepción

\begin{tabular}{|lll|l|}
\hline $\begin{array}{l}\text { Percepción desde el contexto de la } \\
\text { psicología }\end{array}$ & & Percepción desde el contexto socio- \\
& & & \\
& & & \\
\hline Actividad sensorial & & Asignación de significados & \\
& & & \\
\hline
\end{tabular}




\begin{tabular}{|l|l|}
\hline En ella intervienen: & $\begin{array}{l}\text { El núcleo de significación se halla en la esfera } \\
\text { social y colectiva como eje que estructura el } \\
\text { Flujo de comunicación } \\
\text { Categorización } \\
\text { Juicio motivado por la interacción de lo } \\
\text { individual y lo social } \\
\text { conocimiento. } \\
\text { La percepción social enfatiza en la comprensión } \\
\text { del mundo y se construye con base en } \\
\text { intercambios sociales de información. } \\
\text { Conjunto de comprensiones y sensibilidades de } \\
\text { una sociedad sobre su ambiente natural; } \\
\text { proceso social de asignación de significados a } \\
\text { los elementos de un determinado entorno en } \\
\text { una organización. }\end{array}$ \\
\hline
\end{tabular}

Fuente: Elaboración propia con base en revisiones teóricas de la concepción de la percepción.

\section{La organización}

Las definiciones $y$

clasificaciones del término

organización son amplias y variadas.

Para Sheldon (1986), la organización

es el proceso de combinar el trabajo

que los individuos o grupos deben

efectuar con los elementos

necesarios para su ejecución, de tal

manera que las labores ejecutadas sean los mejores medios para la aplicación eficiente, sistemática, positiva y coordinada de los esfuerzos disponibles. Terry (1985) la asume como el arreglo de las funciones que se estiman necesarias para lograr un objetivo, como una indicación de la autoridad y como la responsabilidad asignada a las personas que tienen a su cargo la ejecución de las funciones 
respectivas. Para Etzioni (1965), es un organismo con partes y funciones diversas, que tienen un fin común y para cuyo logro, cada persona desarrolla una acción diversa y complementaria con el objetivo de alcanzar metas específicas.

\section{La estrategia y el cambio como fenómenos al interior de la organización}

Existe una gran variedad de opiniones sobre el concepto de estrategia. Ohmae (1988), por ejemplo, manifiesta que "una estrategia es la manera en que una empresa se esfuerza por distinguirse, en forma positiva, de sus competidores, empleando sus puntos relativamente fuertes para lograr mejor satisfacción de las necesidades del cliente".
Para Porter (1998), la estrategia empresarial es la búsqueda sistemática y deliberada de acciones que permitan y acentúen el desarrollo de la ventaja competitiva de una organización con el objetivo de hacerla crecer y de minimizar los efectos de la competencia. En esta investigación, se considera a la estrategia como el conjunto de habilidades y recursos con los cuales una organización enfrenta de manera exitosa los desafíos que el medio externo le plantea. Así, el establecimiento de estrategias está relacionado con el medio ambiente externo y, en específico, con el sector al cual pertenece una organización.

A tono con lo anterior, las universidades deben diseñar sus estrategias teniendo en cuenta su sector, sus particularidades y 
diferencias con el ámbito empresarial común. Esto requiere de la combinación de actividades que tengan en cuenta su componente académico y de formación, pero sin descuidar elementos como la supervivencia, sostenibilidad y posicionamiento en el medio.

El cambio, por su parte, se asume como la diferencia en forma, calidad o estado, a lo largo del tiempo, de la alineación de la organización con su entorno. Van de Ven \& Poole (1995) y Rajagolapan \& Spreitzer (1997) lo delimitan, estableciendo que las modificaciones de alineación en la organización con su entorno abarcan transformaciones en el contenido de la estrategia de la empresa, tales como su ámbito, distribución de recursos, ventajas competitivas, sinergia, e intercambios en el entorno externo y en la organización.

En la universidad, los estudios organizacionales han tenido un modelo particular. A diferencia de otras organizaciones, los cambios y transformaciones en sus niveles de gestión no han sido suficientes, a pesar de que la expansión de las matrículas sufrida en toda Latinoamérica motivó la búsqueda de nuevas y mejores formas de gestión.

\section{Universidades}

como

\section{anarquías organizadas y sistemas}

\section{flojamente acoplados}

Para Cohen, March \& Olsen (1972), las universidades pueden ser consideradas como anarquías organizadas, debido a unas características particulares, tales como: 
- Fluida participación de sus miembros en los procesos decisorios: a diferencia de otro tipo de organizaciones, las universidades se caracterizan por la acentuada participación de las personas en la toma de decisiones. La democracia juega un papel determinante en muchos de los procesos al interior de estas, con la finalidad de que las decisiones sean legitimadas por la comunidad. No obstante, la participación de los miembros es compleja, porque aspectos como la posición jerárquica, las ideas, las preferencias, la cantidad de trabajo y tiempo dedicados a la organización cambian en el tiempo y en el espacio.

\section{- Ambigüedad en las}

preferencias y percepciones: en las universidades, las personas trabajan para variados fines, los cuales, en ocasiones, resultan contradictorios. Por ejemplo, se realizan con frecuencia procesos que por sí solos reñirían con algunos fines de la propia universidad.

- Dificultad para conectar entradas y salidas de insumos en sus procesos internos: Para Rousseau (1979), la tecnología de una anarquía organizada es difícil de definir y de caracterizar, debido a procesos poco entendibles para los integrantes, quienes terminan actuando por ensayo y error.

- La universidad como sistema flojamente acoplado. Según Weick (1976), en una organización existe flojo acoplamiento cuando las decisiones, creencias y soluciones no están directamente relacionadas con los problemas que se presentan en su interior. Prácticas como la 
acentuación de la descentralización y

la delegación han acentuado el flojo acoplamiento, pues ellas permiten a cada subordinado recrear sus propios objetivos, creencias, información e identidades en su propia unidad.

- Fuerzas contradictorias y dualidad entre orden y desorden: hay fuerzas que tienden hacia la estabilidad, como la planeación y el control; pero otras, como la innovación y la experimentación, llevan hacia la inestabilidad. También hay fuerzas híbridas que coexisten y se contradicen al interior de una anarquía organizada.

- Equilibrio y desequilibrio: tradicionalmente, en el análisis organizacional, el equilibrio es un estado deseado, al cual las fuerzas tienden a converger, y los desequilibrios momentáneos son corregidos por una dinámica que invariablemente regresa al estado inicial.

$$
\text { - Imposibilidad de }
$$

predictibilidad y direccionalidad: desde el paradigma de las anarquías organizadas, la planeación a largo plazo es literalmente imposible, por lo tanto, en estos casos, la evolución de la organización no puede predecirse.

El modelo del cubo de basura como herramienta de toma de decisiones en una anarquía organizada

Cohen, March \& Olsen (1972) determinaron que la toma de decisiones en empresas consideradas como anarquías organizadas, se dificulta porque las preferencias poco claras y difíciles de definir y de observar, generan situaciones imposibles de modelar 
según las teorías racionales y por el constreñimiento en tiempo y recursos

ante la necesidad de realizar otras tareas dentro de la misma organización. A partir de estas premisas, Cohen (1972) crea el modelo llamado Bote de Basura -Garbage Can Model- como herramienta para modelar la toma de decisiones.

La estructura conceptual de este modelo se explica básicamente a partir de las siguientes premisas:

- Los problemas son todo aquello que le preocupa a los miembros dentro y fuera de la organización.

- Las soluciones son el producto de alguien, y resuelven un problema específico; su naturaleza es ambigua puesto que sólo hay soluciones exactas para problemas bien definidos.

- Los participantes son aquellos miembros de la organización que entran $y$ salen en el proceso de decisión.

\section{El neoinstitucionalismo y las}

\section{universidades}

El neoinstitucionalismo es una corriente de pensamiento, cuya idea central pregonaba que las características del contexto de una institución son un factor fundamental para la explicación de sus comportamientos. Si bien las universidades han sido catalogadas como la muestra más fehaciente de una anarquía organizada, se corresponden con lo planteado por el nuevo institucionalismo sociológico, ya que pertenecen a un campo organizacional definido y conformado 
por un gran conjunto y en sus comunidades se institucionalizan reglas, creencias y significados compartidos. El modelo organizacional de las universidades coincide en algunos de sus elementos con el nuevo institucionalismo político promulgado por March \& Olsen (1989), en el sentido de que en su interior se siguen reglas, procedimientos y estrategias alrededor de las cuales se articula su vida y detrás de las cuales subyacen creencias y paradigmas.

\section{RESULTADOS}

En este artículo son presentados los resultados preliminares de la investigación, los cuales reflejan la percepción de dos de los grupos que han sido indagados con relación al tema-directivos de programas y pares académicos-, a quienes les fue aplicada una entrevista semi estructurada que mide su percepción respecto a los factores que conforman el modelo de acreditación por alta calidad. Pero, antes de continuar, es importante conocer los factores que contempla el proceso de acreditación por alta calidad vigente, los cuales son presentados en la Tabla 2.

Tabla 2. Factores que integran el modelo de acreditación por alta calidad en Colombia

\begin{tabular}{|l|l|l|}
\hline Misión y proyecto & Grado de Conocimiento de la misión y del PEI \\
Nivel de apropiación de la misión y del PEI & \\
\hline
\end{tabular}

Desarrollo Gerencial, Vol. 5-2. No. 2-pp. 21-54 julio-Diciembre Universidad Simón Bolívar-Barranquilla - Colombia, ISSN: 2145-5147 .http://portal.unisimonbolivar.edu.co:82/rdigital/desarrollogerencial/index.php/desarrollogerencial/issue/archive 


\begin{tabular}{|c|c|c|}
\hline$F$ & $\begin{array}{l}\text { Procesos } \\
\text { académicos }\end{array}$ & $\begin{array}{l}\text { Pertinencia del Currículo } \\
\text { Niveles de Flexibilidad } \\
\text { Niveles de interdisciplinariedad } \\
\text { Metodologías } \\
\text { sistemas de evaluación de estudiantes }\end{array}$ \\
\hline O & $\begin{array}{l}\text { Visibilidad } \\
\text { nacional e } \\
\text { internacional del } \\
\text { programa }\end{array}$ & $\begin{array}{l}\text { Mejoramiento de la inserción del programa en el contexto } \\
\text { internacional } \\
\text { Número de convenios } \\
\text { Grado de movilidad } \\
\text { Pasantías } \\
\text { Visitantes internacionales }\end{array}$ \\
\hline $\mathrm{S}$ & $\begin{array}{l}\text { Investigación e } \\
\text { innovación }\end{array}$ & $\begin{array}{l}\text { Publicaciones } \\
\text { Nivel de los grupos de investigación } \\
\text { Ponencias } \\
\text { Logro de patentes o de licencias } \\
\text { Pertenencia de docentes y estudiantes a grupos y proyectos }\end{array}$ \\
\hline & $\begin{array}{l}\text { Bienestar } \\
\text { universitario }\end{array}$ & $\begin{array}{l}\text { Niveles de permanencia } \\
\text { Niveles de deserción } \\
\text { Programas de retención } \\
\text { Programas y servicios de bienestar } \\
\text { Atención psicosocial al estudiante }\end{array}$ \\
\hline & $\begin{array}{l}\text { Organización y } \\
\text { gestión }\end{array}$ & $\begin{array}{l}\text { Sistemas de comunicación } \\
\text { Sistemas de gestión }\end{array}$ \\
\hline & $\begin{array}{l}\text { Recursos físicos y } \\
\text { financieros }\end{array}$ & $\begin{array}{l}\text { Recursos físicos } \\
\text { Salones } \\
\text { Bibliotecas } \\
\text { Computadores y equipos }\end{array}$ \\
\hline
\end{tabular}

Desarrollo Gerencial, Vol. 5-2. No. 2-pp. 21-54 julio-Diciembre Universidad Simón Bolívar-Barranquilla - Colombia, ISSN: 2145-5147 .http://portal.unisimonbolivar.edu.co:82/rdigital/desarrollogerencial/index.php/desarrollogerencial/issue/archive 


\begin{tabular}{|l|l|}
\hline & $\begin{array}{l}\text { Presupuesto del programa } \\
\text { Espacios de recreación } \\
\text { Recursos de apoyo para eventos, congresos y seminarios }\end{array}$ \\
\hline
\end{tabular}

Fuente: elaboración propia con base en los factores de acreditación por alta calidad del Consejo Nacional de Acreditación de Colombia.

La entrevista general está Los resultados presentados a conformada por ocho preguntas de continuación sintetizan las respuestas libre respuesta, que permitieron la de 4 expertos en el tema, consultados generación de un diálogo extenso y por su calidad de pares académicos ${ }^{1}$ fluido con cada uno de los $y$ de 8 directivos de programas entrevistados. En el marco del académicos que han sido sometidos presente artículo, fundamentalmente al proceso de acreditación, en se toman como base las respuestas a universidades colombianas. las siguientes dos preguntas: ¿Los factores y características que evalúa el modelo son los necesarios, precisos y suficientes para determinar su calidad?, y ¿Qué elementos adicionaría o eliminaría con respecto al proceso de acreditación por alta calidad de programas actualmente aplicado en Colombia? 
A continuación, se presentan las respuestas dadas por los 12 expertos Tabla 3. Primera pregunta y respuestas de a la primera pregunta (Tabla 3). Estas expertos

se presentan en forma indistinta

porque ambos grupos son igualmente idóneos para emitir esta información.

\begin{tabular}{|l|l|}
\hline entrevistado & $\begin{array}{l}\text { ¿Los factores y características que evalúa el modelo son los necesarios, } \\
\text { precisos y suficientes para determinar su calidad? }\end{array}$ \\
\hline Experto 1 & $\begin{array}{l}\text { Los factores y elementos son los suficientes, pero necesitan más desarrollo. } \\
\text { cada una, el diferencial educativo que las caracteriza y otros aspectos que las } \\
\text { identifican, podría decir que los factores, características e indicadores, no } \\
\text { son todos necesarios, ni precisos, ni suficientes. Sería muy complejo el no } \\
\text { tener una herramienta única de verificación, mas, le corresponde al par } \\
\text { académico el tener la capacidad para agregar valor a la verificación teniendo en } \\
\text { cuenta la herramienta de verificación versus la singularidad interna y externa del } \\
\text { programa universitario que está siendo objeto de verificación, siendo, el par, } \\
\text { objetivo con la realidad de las instituciones. }\end{array}$ \\
\hline
\end{tabular}




\begin{tabular}{|c|c|}
\hline Experto 2 & $\begin{array}{l}\text { Si bien se busca con el proceso de acreditación: } \\
\text { a- Ser un instrumento mediante el cual el Estado da fe pública de la calidad de } \\
\text { los programas de educación superior; se puede decir que es eficaz en cuanto } \\
\text { crezca rápidamente el número de programas acreditados en el país. } \\
\text { b- Brindar información confiable a los usuarios del servicio educativo del nivel } \\
\text { superior; se puede decir que es justo en cuanto se encuentre la información } \\
\text { fidedigna en el SNIES. } \\
\text { c- Propiciar la idoneidad y la solidez de programas académicos de educación } \\
\text { superior; se puede decir que es equitativo en cuanto haya un compromiso ético } \\
\text { en la verificación de las condiciones. } \\
\text { d- Promover en las instituciones la verificación del cumplimiento de su misión, } \\
\text { sus propósitos y sus objetivos en el marco de la Constitución y la Ley, y de } \\
\text { acuerdo con sus propios estatutos; se puede decir que es objetivo en cuanto la } \\
\text { verificación sea imparcial y sin preconceptos creados. }\end{array}$ \\
\hline Experto 3 & $\begin{array}{l}\text { Los sucesivos cambios de la taxonomía de factores del modelo del CNA ponen } \\
\text { de manifiesto que la definición operacional de la "alta calidad académica" de los } \\
\text { programas y las instituciones, mediante factores y características, está en } \\
\text { función de las prescripciones del CESU y el CNA, en torno a un modelo } \\
\text { único de universidad que se ajustan periódicamente a la luz de las políticas } \\
\text { públicas nacionales e internacionales y las interpretaciones de las demandas de } \\
\text { la globalización. }\end{array}$ \\
\hline Experto 4 & $\begin{array}{l}\text { Sí, dado que contemplan las dimensiones, características y elementos } \\
\text { suficientes para realizar una evaluación amplia e integral. Yo considero que son } \\
\text { suficientes. }\end{array}$ \\
\hline Experto 5 & $\begin{array}{l}\text { Pienso que sí, porque contribuyen a que los procesos académico-administrativos } \\
\text { que esto implica, sean más pertinentes y con una alta relevancia social que } \\
\text { supone ambientes educativos más heterogéneos y flexibles, en perspectiva de }\end{array}$ \\
\hline
\end{tabular}

Desarrollo Gerencial, Vol. 5-2. No. 2-pp. 21-54 julio-Diciembre Universidad Simón Bolívar-Barranquilla - Colombia, ISSN: 2145-5147 .http://portal.unisimonbolivar.edu.co:82/rdigital/desarrollogerencial/index.php/desarrollogerencial/issue/archive 


\begin{tabular}{|c|c|}
\hline & $\begin{array}{l}\text { responder adecuadamente a los requerimientos formativos y de investigación de } \\
\text { los respectivos entornos. }\end{array}$ \\
\hline Experto 6 & $\begin{array}{l}\text { Todo lo que se pide, son tantas cosas que a veces no se alcanza a cumplir y } \\
\text { en su gran mayoría muchos se inventan. }\end{array}$ \\
\hline Experto 7 & $\begin{array}{l}\text { Considero que sí, pero que es necesario el apoyo económico por parte del } \\
\text { estado hacia las universidades pequeñas, debido a que la calidad cuesta y no } \\
\text { siempre existe presupuesto suficiente. }\end{array}$ \\
\hline Experto 8 & $\begin{array}{l}\text { La acreditación de los programas académicos, hace más eficiente los procesos } \\
\text { de gestión de la calidad. Porque si una institución no tiene implementado un } \\
\text { eficiente sistema de gestión de la calidad de sus procesos misionales y de apoyo, } \\
\text { no se puede acreditar e incluso si esos procesos no están certificados. }\end{array}$ \\
\hline Experto 9 & os factores son insuficientes, ambiguo \\
\hline Experto 10 & $\begin{array}{l}\text { En cuanto a lo de eficaz, es cuando los objetivos de mejoramiento y calidad en la } \\
\text { prestación del servicio se alcanzan, sin embargo, es posible encontrar programas } \\
\text { acreditados que no cumplen con estas condiciones; lo de justo y equitativo, se } \\
\text { observa que algunas universidades cuentan con respaldo particular para lograr la } \\
\text { acreditación, y en cuanto a lo objetivo, se requiere de mucho profesionalismo y } \\
\text { responsabilidad para llevar a cabo un proceso objetivo, no obstante se observa } \\
\text { que son muy pocos los pares que están inmersos en estos procesos, algunos } \\
\text { incluso se dan el lujo de sin terminar una evaluación aceptan otras, entonces } \\
\text { dónde está lo objetivo. }\end{array}$ \\
\hline Experto 11 & $\begin{array}{l}\text { Si son suficientes, es más son demasiados y hacen que el proceso sea largo, } \\
\text { confuso y demasiado dispendioso. }\end{array}$ \\
\hline Experto 12 & $\begin{array}{l}\text { Son suficientes. El problema del proceso radica en que hay demasiada } \\
\text { información que suministrar y en que los factores y el proceso mismo no permiten } \\
\text { contextualizar }\end{array}$ \\
\hline
\end{tabular}

Fuente: Respuestas de expertos a la entrevista aplicada. 
En síntesis, la mayoría de los expertos representados en 7 de los 12 entrevistados, consideran que los factores $\quad y \quad$ características establecidos son suficientes, incluso algunos piensan que son demasiados, lo cual incide en el cansancio y en ocasiones la confusión, al responder a tan múltiples interrogantes. Pero también hay algunos que piensan que el modelo podría reducirse y ser más preciso a la hora de interrogar sobre la calidad.

Los expertos consideran que
los sucesivos cambios de la
configuración de los factores del
modelo del Consejo Nacional de
Acreditación, ponen de manifiesto
que la definición operacional de la
"alta calidad académica" de los
programas y las instituciones,

mediante factores y características, está más en función de las prescripciones de varios organismos nacionales que ajustan periódicamente el modelo y sus factores a la luz de las políticas públicas nacionales e internacionales, y según las interpretaciones de las demandas de la globalización, tales como el mismo Consejo Nacional de Acreditación-CNA, y como el Consejo Nacional de Educación SuperiorCESU.

Entre los 12 entrevistados, hay una sensación generalizada de que los factores son demasiados y descontextualizados, por lo que es difícil responder a ellos y esta situación hace que el proceso sea descontextualizado y poco objetivo.

La segunda pregunta, cuyas respuestas se presentan en la Tabla 
4, hace referencia a qué elementos

se debería adicionar o eliminarse del

proceso de acreditación por alta calidad de programas, actualmente aplicado en Colombia. Los expertos

opinan:

Tabla 4. Segunda pregunta y respuestas de expertos

\begin{tabular}{|c|c|}
\hline entrevistado & $\begin{array}{l}\text { ¿Qué factores adicionaría o eliminaría con respecto al proceso de } \\
\text { acreditación por alta calidad de programas actualmente aplicado en } \\
\text { Colombia? }\end{array}$ \\
\hline Experto 1 & $\begin{array}{l}\text { Adicionaría indicadores precisos para valorar la calidad en programas de nivel } \\
\text { técnico y tecnológico realmente ajustados a la realidad nacional. }\end{array}$ \\
\hline Exp & $\begin{array}{l}\text { El elemento mediático de la verificación de los pares es una debilidad del proceso; } \\
\text { si bien las universidades con sus programas respaldan con un documento su } \\
\text { quehacer educativo, al evidenciarlo en la visita, se quedan cortos, ya sea por falta } \\
\text { de tiempo o efectividad en las reuniones y presentaciones, y los pares pierden de } \\
\text { vista su labor verificadora del proceso, para pasar a una labor fiscalizadora } \\
\text { mediática, lo que al momento de la visita puede resultar perjudicial para el } \\
\text { programa universitario. } \\
\text { Efectivamente, si bien para el sector productivo, el obtener la certificación de } \\
\text { ICONTEC es importante para la competitividad, asimismo, la acreditación de los } \\
\text { programas académicos en la universidad le significa nacional e internacionalmente } \\
\text { el reconocimiento dentro de la comunidad académica; a la vez que mejora los } \\
\text { procesos de enseñanza-aprendizaje y los procesos de gestión. }\end{array}$ \\
\hline Experto 3 & $\begin{array}{l}\text { Así se insista en simbolizar el proceso de autoevaluación con fines de acreditación } \\
\text { como un proceso auto-referenciado porque el modelo da libertad para ponderar los } \\
\text { factores, las características y los indicadores, las taxonomías de los mismos }\end{array}$ \\
\hline
\end{tabular}




\begin{tabular}{|c|c|}
\hline & $\begin{array}{l}\text { corresponden a un prototipo o ideal institucional predefinido, correlacionado con las } \\
\text { instituciones de "élite" bajo el supuesto de perfecta movilidad social, desconociendo } \\
\text { que el modelo de desarrollo desigual de las regiones del país es determinante de } \\
\text { un mosaico institucional, oficial y privado, que responde a las condiciones } \\
\text { socioeconómicas y culturales de la población, de modo que las IES mas que operar } \\
\text { como un canal para la movilidad social operan como un sistema de válvulas. En } \\
\text { este sentido debería posibilitarse un sistema diferenciado de reconocimiento de } \\
\text { grados progresivos de calidad, tal como el que opera en los mercados financieros. }\end{array}$ \\
\hline Experto 4 & $\begin{array}{l}\text { Trabajar en un proceso permanente de formación de pares, creo que en el país } \\
\text { tenemos trastocado el concepto de par y lo hemos convertido en jueces. Esto } \\
\text { aplica para el CNA. } \\
\text {-Las visitas son tribunales y no espacios académicos "tranquilos" Esto se debe } \\
\text { cambiar, para las instituciones se ha vuelto un lío atender visitas de registro } \\
\text { calificado y acreditación. } \\
\text {-El MEN tiene grandes problemas de gestión institucional que se traduce en } \\
\text { tiempos muy largos de estos procesos }\end{array}$ \\
\hline Experto & $\begin{array}{l}\text { Considero que, definitivamente, los procesos de acreditación tanto institucional } \\
\text { como de sus programas académicos, promueve en las Instituciones de Educación } \\
\text { Superior-IES, el mejoramiento de la calidad de la educación superior, por ende, de } \\
\text { idoneidad y solidez de las instituciones. En esa forma, todos los factores son } \\
\text { importantes porque incentivan a la comunidad académica a reconocer sus } \\
\text { realizaciones y porque garantizan a la sociedad un servicio público de educación } \\
\text { con suficiente calidad. Por otro lado, ofrece credibilidad de los procesos } \\
\text { académico-administrativos y sus resultados. Dado que la evaluación que se realiza } \\
\text { en los procesos de acreditación de alta calidad, hace explicitas las condiciones de } \\
\text { funcionamiento de las IES, y pone en evidencia sus fortalezas y debilidades, es } \\
\text { importante, que dicho proceso que realiza el Consejo Nacional de Acreditación- }\end{array}$ \\
\hline
\end{tabular}




\begin{tabular}{|c|c|}
\hline & $\begin{array}{l}\text { CNA, esté en permanente revisión y ajuste, que sea muy dinámico, que se } \\
\text { socialice con toda la comunicad académica, lo cual permite que su } \\
\text { retroalimentación sea permanente y concertada. } \\
\text { Por otro lado, considero que debe darse más articulación entre el Ministerio de } \\
\text { Educación Nacional-MEN, y sus tres órganos asesores y consultores, como son el } \\
\text { Consejo Nacional de Educación Superior-CESU, el Consejo Nacional de } \\
\text { Acreditación-CNA, y la Comisión Nacional Intersectorial para el Aseguramiento de } \\
\text { la Calidad de la Educación Superior-CONACES; para que no se dé diversidad de } \\
\text { criterios sobre un mismo asunto, como en muchos casos ocurre, donde se } \\
\text { evidencia conceptos contradictorios. }\end{array}$ \\
\hline Exp & $\begin{array}{l}\text { Bueno, esa pregunta si sería bueno que se le hiciera al mismo MEN (Ministerio de } \\
\text { Educación Nacional). Yo pienso que con esas preguntas, y esa manera de } \\
\text { conducir y hacer la autoevaluación no se alcanza a medir lo necesario para } \\
\text { acreditar un programa. Eso es un medio vulnerable que se puede manipular y, } \\
\text { bueno, respeto esta parte pero no la comparto. }\end{array}$ \\
\hline Exp & $\begin{array}{l}\text { Los factores y características utilizados actualmente sirven para lo que se quiere, } \\
\text { pero los procesos de calidad son dinámicos, por lo que esos factores y } \\
\text { características pueden cambiar con el tiempo. Es decir, no pueden ser eternos. }\end{array}$ \\
\hline Experto 8 & $\begin{array}{l}\text { Agregaría que el proceso de autoevaluación con fines de acreditación, } \\
\text { autorregulación y mejoramiento de los programas ofrecidos por las instituciones de } \\
\text { educación superior, no debe ser voluntaria, sino obligatoria bajo el modelo del } \\
\text { CNA, porque sólo así se lograría mejorar la calidad de la educación en el país. Con } \\
\text { los nuevos lineamientos exigidos por el CNA y la ampliación de los factores, las } \\
\text { exigencias son mayores, para lograr no sólo la excelencia académica de los } \\
\text { programas, sino también la acreditación institucional. }\end{array}$ \\
\hline Experto 9 & $\begin{array}{l}\text { - } \quad \text { Separaría las IES, de acuerdo a la Ley 30/1992 } \\
\text { - } \quad \text { Clasificaría las IES de diferentes formas: por tamaño, presupuesto, }\end{array}$ \\
\hline
\end{tabular}

Desarrollo Gerencial, Vol. 5-2. No. 2-pp. 21-54 julio-Diciembre Universidad Simón Bolívar-Barranquilla - Colombia, ISSN: 2145-5147 .http://portal.unisimonbolivar.edu.co:82/rdigital/desarrollogerencial/index.php/desarrollogerencial/issue/archive 


\begin{tabular}{|c|c|}
\hline número de alumnos, región, especialidades, procedencia, etc. \\
$-\quad$ Agruparía los programas de acuerdo con su especialidad, pues eso \\
facilitaría la evaluación de sus aspectos específicos. \\
$-\quad$ Establecería estándares para poder comparar, alcanzar, superar, etc. \\
$-\quad$ Incluiría una evaluación más profunda de la parte administrativa y de la \\
gestión del programa, que actualmente es una de las mayores \\
debilidades que tiene el proceso de acreditación. \\
Contextualizaría la ubicación de los programas, pues son muy distintas \\
las necesidades de acuerdo con la región donde estén ubicados y a la \\
institución a la que pertenecen, entre otras cosas. \\
Propondría una evaluación de tecnología acorde con las circunstancias \\
actuales de la globalización. \\
Sistematizaría los procesos de autoevaluación. \\
Diseñaría un sistema de información que permitiera actualización, \\
control y mayor efectividad en los procesos inherentes a la acreditación \\
tanto institucional como de programas. \\
Implementaría capacitación permanente a todos los actores de estos \\
procesos
\end{tabular}




\begin{tabular}{|c|c|}
\hline Experto 10 & $\begin{array}{l}\text { El proceso de verificación debe ser continuo, es decir, que la institución y el } \\
\text { programa deberían trabajar siempre como lo hacen cuando llegan los pares } \\
\text { académicos, además, implementar otros mecanismos de seguimiento que permitan } \\
\text { evidenciar que realmente la institución presenta cambios en cuanto al } \\
\text { mejoramiento y la calidad del servicio, en caso contrario suspender la acreditación } \\
\text { obtenida. } \\
\text { Debería ser así, pero sin embargo, solo se da en un mínimo porcentaje, puesto que } \\
\text { para algunas instituciones es relativamente fácil acceder a la acreditación de sus } \\
\text { programas, aun si presentan deficiencias en la prestación de los servicios. }\end{array}$ \\
\hline Experto 11 & $\begin{array}{l}\text { Más que agregar o quitar factores, revisaría el proceso y su estructura y haría una } \\
\text { diferencia partiendo de las características de las universidades y programas } \\
\text { visitados, de sus particularidades, incluso separaría el proceso por disciplinas para } \\
\text { hacer más equitativo y transparente el proceso. }\end{array}$ \\
\hline Experto 12 & $\begin{array}{l}\text { No agregaría nada ni quitaría: contextualizaría de acuerdo a la IES (Institución de } \\
\text { Educación superior). }\end{array}$ \\
\hline
\end{tabular}

Fuente: Respuestas de expertos a la entrevista aplicada

Desarrollo Gerencial, Vol. 5-2. No. 2-pp. 21-54 julio-Diciembre Universidad Simón Bolívar-Barranquilla - Colombia, ISSN: 2145-5147 .http://portal.unisimonbolivar.edu.co:82/rdigital/desarrollogerencial/index.php/desarrollogerencial/issue/archive 
En general, los informantes

consideran que los pares

académicos son un elemento que le

da inmensa subjetividad al proceso

y, por lo tanto, su papel debería ser

revisado. En relación con los

factores y características que se

evalúan en una acreditación, se han

reunido las variadas sugerencias

sobre aspectos que a juicio de los

expertos deberían ser revisados, así:

Aspectos generales:

- Contextualizar la medición de

la calidad de acuerdo con las

características de la

Universidad o programa

académico evaluado.

- Clasificar las instituciones de educación superior según sus características.

- Agrupar los programas según su especialidad.
- Contextualizar la ubicación de los programas, pues sus necesidades son muy distintas de acuerdo a su ubicación.

- Sistematizar los procesos de autoevaluación.

- Diseñar un sistema de información que permita actualización, control y mayor efectividad en los procesos institucionales y de programas.

- Implementar sistemas de capacitación permanente a todos los actores de estos procesos.

- Definir un glosario y lenguaje común para contextualizar a los actores con el proceso.

- Definir parámetros claros que permitan establecer la manera en que cumple con el encargo social. 
Por otra parte, las inquietudes expresadas por los expertos se han agrupado así:

- Establecer estándares que permitan comparar, alcanzar o superar ciertos aspectos, como los niveles de internacionalización, entre otros.

- Incluir una evaluación profunda de la parte administrativa del programa.

- Redefinir los parámetros de internacionalización.

- Establecer mecanismos para medir la efectividad del estatuto docente.

- Integrar de una forma más clara al proceso la información de los egresados y su aporte a la sociedad.

- Medir de manera efectiva, entre otros, los vínculos del programa con su entorno.
- Incorporar el cumplimiento y alcance de los objetivos del Bienestar Social Universitario.

De acuerdo con las respuestas emitidas por los informantes, fue evidente que la participación en la aplicación del modelo de autoevaluación con fines de acreditación, les exigió un alto grado de involucramiento con el proceso; entre las respuestas dadas, resalta que la vivencia del proceso fue difícil y estresante.

Como conclusión general, se puede afirmar que la percepción de los expertos frente a los factores que conforman el actual modelo de acreditación de programas académicos en Colombia es de preocupación, debido a lo extenso del modelo y de los factores que lo conforman. En general, se opina que el modelo está 
descontextualizado, y que incluye demasiados y muy extensos factores.

Existe consenso en cuanto a la necesidad de reducir y contextualizar la aplicación del modelo, teniendo en cuenta las particularidades propias de la universidad o programa, ya que no todos poseen las mismas condiciones y características y, por lo tanto, no se someten al proceso en igualdad de condiciones.

\section{REFERENCIAS}

Benney, M., Hughes, E. (1970). Of Sociology and the interview. Journal Sociological Methods: A Sourcebook 4 (75-98).

Cázares, L. (1990). Técnicas actuales de investigación documental. México: Trillas.
Cohen, M., March, J., Olsen, J. (1972). A Garbage can model of organizacional choice. Administrative Science Quarterly. Vol. 17, No 1, pp. $1-25$

Crosby, P. (1979) Quality is Free, New York: Mc Graw Hill.

Croxatto, H. (1988). Algunos Fundamentos y Principios de Acción Universitaria por "et al", Santiago de Chile: Salesianos

Chacón, G. (2006). La Triple Dimensión Cognoscitiva del Término 'Organización' Actualidad Contable FACES. 9 (1). 51-66.

Etzioni, A. (1965). Organizaciones modernas. México: UTEHA. 
Ferraris, M. (2000).La historia de la hermenéutica.

Madrid: Ediciones Akal.

Geerz, C. (1996). La interpretación de las culturas. Barcelona: Gedisa.

Hernández, S., Fernández-Collado, C. Baptista, P. (2008). Metodología de la investigación. México- Mc Graw Hill .

Huerta, J. M. (1980). Los Grupos Focales. Mayagüez. Universidad de Puerto Rico. Husserl E. (1995). La filosofía en la crisis de la humanidad europea. Educación y

Materiales de filosofía. España. Universidad de Valencia.

Kenichi, O. (2007). La Mente del Estratega. México. Mc Graw Hill.
Porter, M. (1988). Ventaja Competitiva (Competitive Advantage), Free press: New York.

León, E., y Zemelman, H. (1997). Subjetividad y umbrales del Pensamiento social. Antrophos: México. March, J. y OlsenJ . (1989).

Rediscovering Institutions, the organizational Basis of Politics.

Free Press: Norway.

March, J., y Simón H., (1958). Teoría de la Organización. Barcelona: Gedisa.

Mertens D.(2005). Research and Evaluation in Education and Psychology: $\quad$ integrating diversity with quantitative and 
qualitative and mixed perspective and integrative

methods. Education, research framework. Academy of and methodology. management Review.

Meyer H. y Rowan, B.. The new institutionalism in education, new York, state University of new York Press.

Ohmae, K. (1988). La Mente del Estratega (Primera ed.). Mc Graw Hill. México,

Pool, V. D. (1955). Explaining development and change in organizations. Academy of Management Review, 30.

Powell, W. (1991). The new institucionalism

in organizational analisis. Chicago: Universidad de Chicago.

Sheldon, O. (1986). La filosofía del management.Orbis.

Solís, P.,y López, V. (2000). El concepto de las anarquías organizadas en el análisis Organizacional en: Revista Contaduría y Administración ( Vol). (19) abril -junio. Pp. $12-25$

Taylor, S. J. y Bogdan, R. (1987). Introducción a los métodos cualitativos de investigación,

Rajagolapan, N., Spreitzer, G. (1997). Toward a theory of Argentina, Paidós. strategic change: a multi-lens 
Terry J., Franklin S. (1985).

lenguaje en sujeto: Existencia

Principios de Administración.

y potencia. México:

México: CESCA.

Anthropos.

Valles, M. (2007). Entrevistas

cuatnitativas. En: Cuadernos

Metodologicos No 32. Madrid.

Van de ven, A., Poole, M.S. (1995).

Explaining development and change in Organizations.

Academy of Management

Review.

Weber, M. (1978). Economy and

Society an Outline of

Interpretative Sociology.

Berkeley: University of

California Press.

Weick, K. E. (1976). Educational

organizations as Loosely

Couples Sistems

:Administrative Science

Quarterly.

Zemelman, H. (1998). Existencia y

conocimiento: El doble 